\title{
On the Autoconvolution Equation and Total Variation Constraints *
}

\author{
Gunter Fleischer $^{\dagger} \quad$ Rudolf Gorenflo $^{\ddagger} \quad$ Bernd HofmanN $^{\S}$ \\ 1991 Mathematics Subject Classifications: \\ 65 J20, 45 G10, 65 R30
}

Keywords:

autoconvolution, ill-posed problem, discretization, constrained least-squares approach, bounded total variation

\begin{abstract}
This paper is concerned with the numerical analysis of the autoconvolution equation $x * x=y$ restricted to the interval $[0,1]$. We present a discrete constrained least squares approach and prove its convergence in $L^{p}(0,1), 1 \leq p<\infty$, where the regularization is based on a prescribed bound for the total variation of admissible solutions. This approach includes the case of non-smooth solutions possessing jumps. Moreover, an adaption to the Sobolev space $H^{1}(0,1)$ and some remarks on monotone functions are added. The paper is completed by a numerical case study concerning the determination of non-monotone smooth and non-smooth functions $x$ from the autoconvolution equation with noisy data $y$.
\end{abstract}

${ }^{*}$ The research was supported by the Deutsche Forschungsgemeinschaft (DFG) Grant Ho 1454/3-2 and by the Alexander von Humboldt Foundation, Bonn.

${ }^{\dagger}$ Faculty of Mathematics, Technische Universität Chemnitz-Zwickau, D-09107 Chemnitz, Germany, email: fleische@mathematik.tu-chemnitz.de

${ }^{\ddagger}$ Department of Mathematics and Computer Science, Free University of Berlin, Arnimallee 2-6, D-14195 Berlin, Germany, email: gorenflo@math.fu-berlin.de

${ }^{\S}$ Faculty of Mathematics, Technische Universität Chemnitz-Zwickau, D-09107 Chemnitz, Germany, email: hofmannb@mathematik.tu-chemnitz.de 


\section{Introduction}

In the paper [8] by GoRENFLo and Hofmann the nonlinear ill-posed autoconvolution equation

$$
\int_{0}^{s} x(s-t) x(t) d t=y(s), \quad 0 \leq s \leq 1
$$

on the finite interval $[0,1]$ has been analyzed. This autoconvolution problem can be written as an operator equation

$$
F(x)=y
$$

with the continuous nonlinear operator $F: D(F) \subset X \rightarrow Y$ defined by

$$
[F(x)](s):=[x * x](s):=\int_{0}^{s} x(s-t) x(t) d t, \quad 0 \leq s \leq 1,
$$

and mapping between Banach spaces $X$ and $Y$ with norms $\|\cdot\|_{X}$ and $\|\cdot\|_{Y}$, respectively, containing real functions on the interval $[0,1]$. In [8] there have been discussed intrinsic properties of the autoconvolution operator $F$ from (1.3) and conditions for its compactness, injectivity and weak closedness, in particular for the Hilbert space $X=Y=L^{2}(0,1)$. As a consequence the general theory of Tikhonov regularization became applicable to equation (1.1). The character of ill-posedness in this equation strongly depends on the solution point $x$ and its local degree of ill-posedness. Applications of the autoconvolution equation arising in physics and in stochastics are also mentioned in [8].

On the other hand, we discussed in a recent paper (cf. [5]) including numerical results the case that $x$ is considered as a function of the space $L^{2}(-\infty, \infty)$ possessing a support supp $x \subset[0,1]$, where the complete data function $[x * x](s)(0 \leq s \leq 2)$ is observable. In such a case Fourier transform techniques are applicable and yield some more insight into the behaviour of the autoconvolution equation. However, the knowledge of $[x * x](s)$ for $s>1$ is not always realistic. Therefore, in the present paper we are going to investigate stable approximate discretized solutions to (1.1), where both the function $x$ to be determined and the data function $y$ that can be measured are restricted to arguments from the interval $[0,1]$.

The approximate solution of the autoconvolution equation (1.1) will be based for $Y:=$ $L^{2}(0,1)$ on the restriction of admissible solutions $x$ to compact subsets of the domain $D(F)$ with prescribed properties. Provided that $F$ is injective the inverse operator $F^{-1}$ becomes continuous. We will show in Section 2 that a compactification of the autoconvolution equation in $X:=L^{p}(0,1)$ can be based on a prescribed upper bound $c$ for the total variation $T(x)$ of 
solutions $x$, which are in addition uniformly bounded below and above by positive constants $a$ and $b$, respectively. This allows us to construct convergent discretized solutions also in the case of non-smooth solutions possessing jumps. In this context, we generalize the well-known descriptive regularization approach using the set of monotone functions uniformly bounded below and above as a compact subset in $L^{p}(0,1), 1 \leq p<\infty$ (cf. Section 4, [13] and [4]). The total variation bound $c$ plays in our consideration the role of a regularization parameter. In Section 3, the ideas of Section 2 are extended to the Sobolev space case $X:=H^{1}(0,1)$. A brief reference to the case of monotone functions is given in Section 4. The paper is completed by a case study presented in Section 5 that illustrates the theoretical assertions of Section 2 . In this case study the behaviour of discretized least-squares solutions to the autoconvolution equation subject to uniform bounds of the total variation is investigated, where both the case of a smooth and of a non-smooth solution are reflected.

\section{Discretizing the Autoconvolution Equation under Total Vari- ation Constraints}

Let us consider the autoconvolution operator (1.3) between the Banach spaces $X:=L^{p}(0,1)$ for fixed $2 \leq p<\infty$ with norm $\|x\|_{L^{p}(0,1)}=\left(\int_{0}^{1}|x(t)|^{p} d t\right)^{1 / p}$ and $Y:=L^{2}(0,1)$. In this context, we define the sets

$$
D_{\varepsilon}^{+}:=\left\{x \in L^{p}(0,1): x(t) \geq 0 \text { a.e. in }[0,1], \varepsilon=\sup \{\tau: x(t)=0 \text { a.e. in }[0, \tau]\}\right\}
$$

and

$$
R_{\varepsilon}^{+}:=\left\{y \in L^{2}(0,1): y(s) \geq 0 \text { a.e in }[0,1], \varepsilon=\sup \{\chi: y(s)=0 \text { a.e. in }[0, \chi]\}\right\}
$$

Then we have the following proposition which, because of $L^{p}(0,1)$ being densely embedded in $L^{2}(0,1)$, follows from [8, Theorem 1 and Lemma 6] and [5, Proposition 2.5]:

Proposition 2.1 The autoconvolution operator $F: L^{p}(0,1) \rightarrow L^{2}(0,1)$ from (1.3) is a continuous nonlinear operator for all $2 \leq p<\infty$. In the restricted case $F: D_{0}^{+} \subset L^{p}(0,1) \rightarrow$ $R_{0}^{+} \subset L^{2}(0,1)$ the operator is injective, but the autoconvolution equation (1.2) is locally illposed in the sense of Definition 2.2 in all points $x \in D_{0}^{+}$. 
Definition 2.2 We call the equation (1.2) locally ill-posed in $x \in D(F)$ if, for arbitrarily small $r>0$ and balls $B_{r}:=\left\{\tilde{x} \in X:\|\tilde{x}-x\|_{X} \leq r\right\}$, there is an infinite sequence $\left\{x_{k}\right\} \subset$ $D(F) \cap B_{r}(x)$ with

$$
\left\|F\left(x_{k}\right)-F(x)\right\|_{Y} \rightarrow 0, \quad \text { but } \quad\left\|x_{k}-x\right\|_{X} \not 0 \quad \text { as } \quad k \rightarrow \infty
$$

Otherwise the equation is called locally well-posed in $x \in D(F)$.

To overcome the difficulties of ill-posedness of a problem under consideration one can restrict the domain $D(F)$ to a subset, which is compact in the Banach space $X$.

For a real function $x(t)(0 \leq t \leq 1)$ we denote by

$$
T(x):=\sup _{0 \leq t_{0}<t_{1}<\ldots<t_{k-1}<t_{k} \leq 1} \sum_{i=1}^{k}\left|x\left(t_{i}\right)-x\left(t_{i-1}\right)\right|
$$

the total variation of the function $x$ on $[0,1]$ and by $T_{S}(x)$ the analogously defined total variation of $x$ on a closed subinterval $S \subset[0,1]$. Note that the supremum in formula (2.4) is to be taken over all possible finite grids of the form $0 \leq t_{0}<t_{1}<\ldots<t_{k-1}<t_{k} \leq 1$ with an arbitrarily chosen integer $k$. We consider, for given positive constants $a, b$ and $c$, where

$$
0<a<b
$$

the domain

$$
D:=\left\{x:[0,1] \rightarrow[a, b], \quad T(x) \leq c, \quad \begin{array}{c}
\mathrm{x} \text { left-continuous for } t \in(0,1] \\
\mathrm{x} \text { right-continuous for } \mathrm{t}=0
\end{array}\right\}
$$

For technical reasons we assume that the lower bound $a$ is strictly positive (see the remark after formula (2.21)). Obviously we have $D \subset L^{p}(0,1)$ for all $1 \leq p<\infty$. The requirement of the left- and right-continuity for the functions $x \in D$ is reasonable, since a function of bounded variation has due to [12, Corollary 2, Chap. VIII, $\S 3]$ only a countable set of discontinuity points, namely jumps. Therefore, the left $\operatorname{limit}_{t \rightarrow t_{0}-0} x(t)$ exists in all points of the interval $(0,1]$. In the continuity points $t_{0}$ this limit coincides with the value $x\left(t_{0}\right)$. In all other points let be the values of $x$ defined by $x\left(t_{0}\right):=\lim _{t \rightarrow t_{0}-0} x(t)$. That means, with respect to $L^{p}(0,1)$-elements we consider the representative, which is left-continuous in every point $t \in(0,1]$. Moreover let $x(0):=\lim _{t \rightarrow 0+0} x(t)$, i.e. we consider no jumps at $t=0$.

Lemma 2.3 The domain $D$ from (2.5) - (2.6) is a compact subset of $L^{p}(0,1), 1 \leq p<\infty$, and we have $D \subset D_{0}^{+}$. 
The proof of compactness of $D$ is based on HeLLY's theorem (cf. e.g. [12, Chap. VIII, §4]). For the proof ideas we refer to [4, Lemma 4.2]. On the other hand, note that Lemma 2.3 is a corollary of Theorem 2.5 in the paper [1] of ACAR and Vogel. Namely, the set $D$ from $(2.5)-(2.6)$ is bounded with respect to the BV-norm

$$
\|x\|_{B V[0,1]}:=\|x\|_{L^{1}[0,1]}+T(x) .
$$

Based on Lemma 2.3 providing compactness the following well-known Lemma of TikHONOV will allow us to prove stability results.

Lemma 2.4 Let $F: D(F) \subset X \rightarrow Y$ be a continuous and injective operator between the Banach spaces $X$ and $Y$ with a compact domain $D(F)$. We denote by $x^{*}$, for given righthand side $y^{*} \in F(D(F))$, the unique solution of the operator equation (1.2). Then for a family of approximate solutions $x_{\eta} \in D(F)$ the convergence of residual norms

$$
\left\|F\left(x_{\eta}\right)-F\left(x^{*}\right)\right\|_{Y} \rightarrow 0 \quad \text { as } \quad \eta \rightarrow 0
$$

implies the convergence of the approximate solutions

$$
\left\|x_{\eta}-x^{*}\right\|_{X} \rightarrow 0 \quad \text { as } \quad \eta \rightarrow 0
$$

A slightly modified version of this theorem and its proof can be found in BAUMEISTER's book [2, p. 18].

In order to obtain numerical approximate solutions, in the sequel we are going to discretize the autoconvolution equation (1.1) - (1.3), where the restriction of $F$ to the compact subset $D$ from $(2.5)-(2.6)$,

$$
F: D \subset L^{p}(0,1) \rightarrow L^{2}(0,1)
$$

is used. Similar to the discretization methods in [7] and [11], where also a total variation constraint is essential, we subdivide the interval $[0,1]$ into $n$ subintervals $I_{i}$ of the uniform length $h:=1 / n$, where

$$
I_{i}:=((i-1) h, i h] \quad(i=1, \ldots, n)
$$

For simplicity we set $T_{i}(x):=T_{[(i-1) h, i h]}(x)$ for $x \in D$. Moreover, let

$$
t_{j}:=\frac{h}{2}+(j-1) h \quad(j=1, \ldots, n)
$$


denote the midpoints and

$$
s_{i}:=i h \quad(i=1, \ldots, n)
$$

the right endpoints of such intervals.

To discretize the nonlinear integral equation (1.1), for all $i, j=1,2, \ldots, n$ the values $x\left(t_{j}\right)$ and $y\left(s_{i}\right)$ will be approximated by some $x_{j}$ and $y_{i}$, respectively. A discrete autoconvolution operator

$$
\underline{F}: \mathbb{R}^{n} \rightarrow \mathbb{R}^{n}
$$

can be defined by

$$
\underline{F}(\underline{x}):=\left(\sum_{j=1}^{i} h x_{i-j+1} x_{j}\right)_{i=1}^{n}, \quad \underline{x}=\left(x_{1}, \ldots, x_{n}\right)^{T} .
$$

In its discrete form the autoconvolution equation then reads as

$$
\underline{F}(\underline{x})=\underline{y}, \quad \underline{y}=\left(y_{1}, \ldots, y_{n}\right)^{T},
$$

or as

$$
\sum_{j=1}^{i} h x_{i-j+1} x_{j}=y_{i}, \quad(i=1, \ldots, n) .
$$

The realistic situation that the given data are noisy can be included. Instead of the exact data $y_{i}$ for the right-hand side we will use perturbed data $\hat{y}_{i}$, where

$$
\|\underline{\hat{y}}-\underline{y}\|_{2} \leq \delta
$$

and $\delta$ is a fixed upper bound for the noise of the data vector $\underline{\hat{y}}=\left(\hat{y}_{1}, \ldots, \hat{y}_{n}\right)^{T}$. Here we have used the scaled Euclidean norm

$$
\|\underline{z}\|_{2}:=\left(\sum_{i=1}^{n} h z_{i}^{2}\right)^{\frac{1}{2}}
$$

for $\underline{z} \in \mathbb{R}^{n}$. For our further investigations we introduce the restriction operators

$$
R: D \subset L^{p}(0,1) \rightarrow \mathbb{R}^{n} \quad \text { and } \quad Q: F(D) \subset L^{2}(0,1) \rightarrow \mathbb{R}^{n}
$$

by

$$
(R(x))_{j}:=x\left(t_{j}\right) \quad(j=1, \ldots, n)
$$

and

$$
(Q(y))_{i}:=y\left(s_{i}\right) \quad(i=1, \ldots, n)
$$


as well as the extension operators $E_{1}: \mathbb{R}^{n} \rightarrow L^{p}(0,1)$ and $E_{2}: \mathbb{R}^{n} \rightarrow L^{2}(0,1)$ by

$$
\left(E_{1}(\underline{x})\right)(t):=x_{j} \quad\left(t \in I_{j}, j=1, \ldots, n\right), \quad\left(E_{1}(\underline{x})\right)(0):=x_{1}
$$

and

$$
\left(E_{2}(\underline{y})\right)(s):=y_{i} \quad\left(s \in I_{i}, i=1, \ldots, n\right), \quad\left(E_{2}(\underline{y})\right)(0):=y_{1} .
$$

We are searching now for an optimal solution vector

$$
\underline{x}^{o p t}=\left(x_{1}^{o p t}, \ldots, x_{n}^{o p t}\right)^{T}
$$

solving the discrete least-squares problem

$$
\|\underline{F}(\underline{x})-\underline{\hat{y}}\|_{2} \rightarrow \min , \quad \text { subject to } \quad \underline{x} \in M,
$$

where $M$ is defined as

$$
M:=\left\{\underline{x} \in \mathbb{R}^{n}: 0<a \leq x_{i} \leq b(i=1, \ldots, n), \quad \sum_{i=1}^{n-1}\left|x_{i+1}-x_{i}\right| \leq c\right\} .
$$

There exist solutions of $(2.20)$, since $M$ is compact in $\mathbb{R}^{n}$ and $\|\underline{F}(\underline{x})-\underline{\hat{y}}\|_{2}: \mathbb{R}^{n} \rightarrow \mathbb{R}^{1}$ is a continuous functional possessing a minimum over $M$. The condition $0<a \leq x_{i} \leq b$ is more restrictive than the discretized version of $x \in D_{0}^{+}$. We require this stronger condition, because we want $M$ to be a compact subset of $\mathbb{R}^{n}$.

For the vectors $\eta:=(\delta, h)^{T}, \underline{x}^{o p t} \in M$ and $\underline{\hat{y}}$ we define the piecewise constant function $x_{\eta} \in D$ by

$$
x_{\eta}(t):=E_{1}\left(\underline{x}^{o p t}\right)(t) \quad(0 \leq t \leq 1) .
$$

and the piecewise constant function $y_{\delta}$ by

$$
y_{\delta}(s):=E_{2}(\underline{\hat{y}})(s) \quad(0 \leq s \leq 1) .
$$

Lemma 2.5 If we define the operator $F_{\eta}: L^{p}(0,1) \rightarrow L^{2}(0,1)$ by the formula

$$
\left[F_{\eta}(x)\right](s):=\sum_{j=1}^{i} \int_{I_{j}} x\left(s_{i}-t\right) x(t) d t \quad\left(s \in I_{i}\right),
$$

then we have the equation

$$
\left\|F_{\eta}(\xi)-\zeta\right\|_{L^{2}(0,1)}^{2}=\|\underline{F}(\underline{\xi})-\underline{\zeta}\|_{2}^{2}
$$

for all $\xi:=E_{1}(\underline{\xi})$, where $\underline{\xi}:=\left(\xi_{1}, . ., \xi_{n}\right)^{T} \in \mathbb{R}^{n}$ and all $\zeta:=E_{2}(\underline{\zeta}) \in L^{2}(0,1)$, where $\underline{\zeta}:=\left(\zeta_{1}, . ., \zeta_{n}\right)^{T} \in \mathbb{R}^{n}$. 


\section{Proof:}

$$
\begin{aligned}
\left\|F_{\eta}(\xi)-\zeta\right\|_{L^{2}(0,1)}^{2} & =\int_{0}^{1}\left(\left[F_{\eta}(\xi)\right](s)-\zeta(s)\right)^{2} d s \\
& =\sum_{i=1}^{n} \int_{I_{i}}\left(\sum_{j=1}^{i} \int_{I_{j}} \xi\left(s_{i}-t\right) \xi(t) d t-\zeta(s)\right)^{2} d s \\
& =\sum_{i=1}^{n} h\left(\sum_{j=1}^{i} h \xi_{i-j+1} \xi_{j}-\zeta_{i}\right)^{2} \\
& =\|\underline{F}(\underline{\xi})-\underline{\zeta}\|_{2}^{2} .
\end{aligned}
$$

This proves the lemma

Lemma 2.6 Let $x \in D$ from (2.5) - (2.6). Then we have the estimation

$$
\left\|F(x)-F_{\eta}(x)\right\|_{L^{2}(0,1)} \leq 2 h b^{2}+2 h b c .
$$

Proof: We write

$$
\left\|F(x)-F_{\eta}(x)\right\|_{L^{2}(0,1)}=\left(\sum_{i=1}^{n} \int_{I_{i}}\left(\int_{0}^{s} x(s-t) x(t) d t-\int_{0}^{s_{i}} x\left(s_{i}-t\right) x(t) d t\right)^{2} d s\right)^{\frac{1}{2}} .
$$

Then we can estimate the expression in the inner parentheses by

$$
\begin{aligned}
& \left|\int_{0}^{s} x(s-t) x(t) d t-\int_{0}^{s_{i}} x\left(s_{i}-t\right) x(t) d t\right| \\
\leq & \left|\int_{s_{i-1}}^{s} x(s-t) x(t) d t\right|+\left|\int_{0}^{s_{i-1}} x(s-t) x(t) d t-x\left(s_{i}-t\right) x(t) d t\right|+\left|\int_{s_{i-1}}^{s_{i}} x\left(s_{i}-t\right) x(t) d t\right| \\
\leq & h b^{2}+\sum_{j=1}^{i-1} \int_{I_{j}}\left|x(s-t)-x\left(s_{i}-t\right)\right||x(t)| d t+h b^{2} \\
\leq & b \sum_{j=1}^{i-1} \int_{I_{j}}\left|x(s-t)-x\left(s_{i}-t\right)\right| d t+2 h b^{2} .
\end{aligned}
$$

Now we substitute $u:=s_{i}-t, d u:=-d t$. For a fixed point $t \in\left(s_{j-1}, s_{j}\right]=I_{j}$ we obtain $u \in\left(s_{i-j}, s_{i-j+1}\right]=I_{i-j+1}$ and in view of $-h \leq s-s_{i} \leq 0$

$$
s-s_{i}+u \in\left(s_{i-j-1}, s_{i-j+1}\right]=I_{i-j} \cup I_{i-j+1} .
$$

Moreover, we can estimate (2.26) by

$$
b \sum_{j=1}^{i-1} \int_{I_{j}}\left|x(s-t)-x\left(s_{i}-t\right)\right| d t+2 h b^{2}
$$




$$
\begin{aligned}
& =b \sum_{j=1}^{i-1} \int_{I_{i-j+1}}\left|x\left(s-s_{i}+u\right)-x(u)\right| d u+2 h b^{2} \\
& \leq h b \sum_{j=1}^{i-1}\left(T_{i-j}(x)+T_{i-j+1}(x)\right)+2 h b^{2} \\
& \leq h b T(x)+h b T(x)+2 h b^{2} \\
& \leq 2 h b c+2 h b^{2} .
\end{aligned}
$$

Finally we substitute this estimation into equation (2.25). This yields the assertion of the lemma

Lemma 2.7 Under the assumptions stated above we have

$$
\left\|F\left(x_{\eta}\right)-F\left(x^{*}\right)\right\|_{L^{2}(0,1)} \leq 4 h b^{2}+6 h b c+2 \delta \rightarrow 0 \quad \text { as } \quad \eta \rightarrow 0 .
$$

Proof (for similar ideas see also [6]): From the triangle inequality we obtain

$$
\left\|F\left(x_{\eta}\right)-F\left(x^{*}\right)\right\|_{L^{2}(0,1)} \leq\left\|F\left(x_{\eta}\right)-F_{\eta}\left(x_{\eta}\right)\right\|_{L^{2}(0,1)}+\left\|F_{\eta}\left(x_{\eta}\right)-y_{\delta}\right\|_{L^{2}(0,1)}+\left\|y_{\delta}-y\right\|_{L^{2}(0,1)} .
$$

The right-hand side of (2.28) consists of three terms which we want to estimate one by one: Due to Lemma 2.6 for the first term it holds

$$
\left\|F\left(x_{\eta}\right)-F_{\eta}\left(x_{\eta}\right)\right\|_{L^{2}(0,1)} \leq 2 h b^{2}+2 h b c \quad\left(x_{\eta} \in D\right) .
$$

To estimate the second term of (2.28) we define $\underline{x}^{*}:=R\left(x^{*}\right)$ as the vector of the function values of the exact solution $x^{*}$ of the autoconvolution equation (1.1) in the midpoints of the intervals $I_{i}$. Since we have $\underline{x}^{o p t}$ as the least-squares solution of $(2.20)$, the residual norm of $\underline{x}^{*}$ cannot be smaller than the residual norm of $\underline{x}^{\text {opt }}$. Furthermore, we can apply Lemma 2.5 with $\xi:=x_{\eta}$ and $\zeta:=y_{\delta}$. This yields

$$
\left\|F_{\eta}\left(x_{\eta}\right)-y_{\delta}\right\|_{L^{2}(0,1)}=\left\|\underline{F}\left(\underline{x}^{o p t}\right)-\underline{\hat{y}}\right\|_{2} \leq\left\|\underline{F}\left(\underline{x}^{*}\right)-\underline{\hat{y}}\right\|_{2} .
$$

Using the identity

$$
F_{\eta}(x)=E_{2}(Q(F(x))) \quad(x \in D)
$$

this allows us to estimate further as follows:

$$
\begin{aligned}
& \left\|\underline{F}\left(\underline{x}^{*}\right)-\underline{\hat{y}}\right\|_{2} \leq\left\|\underline{F}\left(\underline{x}^{*}\right)-Q\left(F\left(x^{*}\right)\right)\right\|_{2}+\left\|Q\left(F\left(x^{*}\right)\right)-\underline{\hat{y}}\right\|_{2} \\
= & \left\|F_{\eta}\left(E_{1}\left(R\left(x^{*}\right)\right)\right)-E_{2}\left(Q\left(F\left(x^{*}\right)\right)\right)\right\|_{L^{2}(0,1)}+\|\underline{y}-\underline{\hat{y}}\|_{2}
\end{aligned}
$$




$$
\begin{aligned}
& =\left(\sum_{i=1}^{n} \int_{I_{i}}\left(\sum_{j=1}^{i} \int_{I_{j}}\left(\tilde{x}\left(s_{i}-t\right) \tilde{x}(t)-x^{*}\left(s_{i}-t\right) x^{*}(t)\right) d t\right)^{2} d s\right)^{\frac{1}{2}}+\delta \\
& \leq\left(\sum_{i=1}^{n} \int_{I_{i}}\left(\sum_{j=1}^{i} \int_{I_{j}}\left|\tilde{x}\left(s_{i}-t\right)\right|\left|\tilde{x}(t)-x^{*}(t)\right|+\left|x^{*}\left(s_{i}-t\right)-\tilde{x}\left(s_{i}-t\right)\right|\left|x^{*}(t)\right| d t\right)^{2} d s\right)^{\frac{1}{2}}+\delta \\
& \leq\left(\sum_{i=1}^{n} \int_{I_{i}}\left(\sum_{j=1}^{i} \int_{I_{j}} 2 b T_{j}\left(x^{*}\right) d t\right)^{2} d s\right)^{\frac{1}{2}}+\delta \leq 2 h b c+\delta,
\end{aligned}
$$

where $\tilde{x}:=E_{1}\left(R\left(x^{*}\right)\right)$. The last inequalities essentially used Lemma 2.5 with $\xi=E_{1}\left(R\left(x^{*}\right)\right)=\tilde{x}$ and $\zeta=E_{2}\left(Q\left(F\left(x^{*}\right)\right)\right)$, respectively. Note that we have $\tilde{x}(t)=x^{*}\left(t_{j}\right)$ for $t \in I_{j}$ and thereby $\left|\tilde{x}(t)-x^{*}(t)\right| \leq T_{j}\left(x^{*}\right)$. Taking into account $\left|y_{i}-\hat{y}_{i}\right| \leq \delta$ and the identity

$$
\left\|E_{2}(\underline{y})\right\|_{L^{2}(0,1)}=\|\underline{y}\|_{2}
$$

which can easily be proved, we hence can estimate the third term of (2.28) as follows (cf. Lemma 2.6):

$$
\begin{aligned}
& \left\|y_{\delta}-y\right\|_{L^{2}(0,1)} \leq\left\|y-E_{2}(Q(y))\right\|_{L^{2}(0,1)}+\left\|E_{2}(Q(y))-y_{\delta}\right\|_{L^{2}(0,1)} \\
= & \left\|F\left(x^{*}\right)-E_{2}\left(Q\left(F\left(x^{*}\right)\right)\right)\right\|_{L^{2}(0,1)}+\left\|E_{2}(Q(y))-E_{2}\left(Q\left(y_{\delta}\right)\right)\right\|_{L^{2}(0,1)} \\
= & \left\|F\left(x^{*}\right)-F_{\eta}\left(x^{*}\right)\right\|_{L^{2}(0,1)}+\left\|Q(y)-Q\left(y_{\delta}\right)\right\|_{2} \leq 2 h b^{2}+2 h b c+\delta .
\end{aligned}
$$

Finally we can add the three terms and obtain by (2.28) the inequality (2.27). Evidently, the right-hand side of (2.27) tends to zero as $h$ and $\delta$ both tend to zero. This proves the lemma

By the result of Lemma 2.7 we can apply Lemma 2.4 to prove in $L^{p}$-spaces the convergence of approximate solutions to the autoconvolution equation under total variation constraints.

Theorem 2.8 Consider the autoconvolution problem (1.1) - (1.3) with $D(F):=D$ from (2.5)-(2.6) and denote by $x^{*} \in D$, for given right-hand side $y^{*} \in F(D(F))$, the unique solution of the autoconvolution equation. Then the family of approximate solutions $x_{\eta}$ according to (2.22) converges to the solution $x^{*}$ of (1.2):

$$
\left\|x_{\eta}-x^{*}\right\|_{L^{p}(0,1)} \rightarrow 0 \quad \text { as } \quad \eta \rightarrow 0 \quad \text { for all } \quad 1 \leq p<\infty
$$

Proof: In the case $p \geq 2$ based on Lemma 2.7 the Lemma 2.4 immediately yields the convergence property (2.29), since the autoconvolution operator $F: D \subset L^{p}(0,1) \rightarrow L^{2}(0,1)$ 
is continuous and injective. Furthermore, $D$ is a compact subset in $L^{p}(0,1)$ because of Lemma 2.3. For $1 \leq p<2$ the norm $\|\cdot\|_{L^{p}(0,1)}$ is 'weaker' than the norm $\|\cdot\|_{L^{2}(0,1)}$. This ensures the convergence condition (2.29) also in this case

By using the method of TIKHONOv regularization in Hilbert spaces $X$ and $Y$ the minimizers $x_{\alpha}$ of the auxiliary extremal problems

$$
\|F(x)-y\|_{Y}^{2}+\alpha\|x\|_{X}^{2} \rightarrow \min , \quad \text { subject to } \quad D(F)
$$

with the regularization parameter $\alpha>0$ are exploited to find stable approximate solutions of an ill-posed operator equation (1.2). The smaller the regularization parameter $\alpha$ is chosen, the 'closer' the original and the auxiliary problem are related, but the more instable and highly oscillating the solution of the auxiliary problem will become. In general, $\alpha$ has to be selected such that an appropriate trade-off between stability and approximation is realized. In our compactification approach using upper bounds $c$ of the total variation the inverse

value $\frac{1}{c}$ plays a comparable role. In fact, if we consider small values $\frac{1}{c}$, then highly oscillating functions with large total variation values are admissible. On the other hand, for small values $c$ the solutions obtained cannot oscillate very much, and the approximate solutions will be computed in a more stable way. However, if $c$ is selected too small, then it may occur that the (unknown) exact solution is not an element of the set $D$. In such a case we would 'overregularize' the autoconvolution equation. By controlling the upper bound $c$ of total variation we are able to suppress oscillations. Compared to the frequently used compactification in $L^{p}$ by using monotonicity constraints and lower and upper bounds for the function values (see Section 4) the approach of this section allows us to handle a more comprehensive class of (also non-monotone) functions. A numerical case study presented in Section 5 will illustrate the theoretical results of this section and some specific effects of the discretized solution of the autoconvolution equation under total variation constraints.

In the case $p=\infty$ we cannot assert convergence under our assumption of bounded total variation. If the solution $x^{*}$ has a jump point, then $\left\|x_{\eta}-x^{*}\right\|_{L^{\infty}(0,1)} \rightarrow 0$ as $\eta \rightarrow 0$ is not true in general.

\section{The Sobolev Space Case}

In [8] it was already mentioned that the operator $F$ of autoconvolution according to (1.3) mapping from $X:=L^{2}(0,1)$ into the space $Y:=L^{2}(0,1)$ is non-compact, but it becomes a 
compact operator if we change the problem to the Sobolev space $X:=H^{1}(0,1) \cong W_{2}^{1}(0,1)$ of functions $x$ with a quadratically integrable generalized derivative $x^{\prime}$ and norm

$$
\|x\|_{H^{1}(0,1)}=\left(\int_{0}^{1}|x(t)|^{2} d t+\int_{0}^{1}\left|x^{\prime}(t)\right|^{2} d t\right)^{1 / 2} .
$$

In both cases the autoconvolution equation is locally ill-posed everywhere. But for compact operators $F$, we have in general a stronger form of ill-posedness. If our pairs of spaces $X$ and $Y$ are Hilbert spaces, following the concept of [8] (see also [9, Sect. 2.2.2]) we can express the local degree of ill-posedness $\mu(0 \leq \mu \leq \infty)$ of the autoconvolution equation in a solution point $x^{*}$ by the decay rate of the singular value sequence $\sigma_{1} \geq \sigma_{2} \geq \ldots \geq \sigma_{i} \geq \ldots>0$ tending to zero as $i \rightarrow \infty$ of the Fréchet derivative $F^{\prime}\left(x^{*}\right)$ in the form

$$
\mu:=\sup \left\{\nu: \sigma_{i}=O\left(i^{-\nu}\right) \text { as } i \rightarrow \infty\right\}
$$

where this linear operator given by $F^{\prime}\left(x^{*}\right) h=2 h * x^{*}$ is compact. Since the compact embedding operator from $H^{1}(0,1)$ into $L^{2}(0,1)$ has a sequence of singular values $\kappa_{1} \geq \kappa_{2} \geq$ $\ldots \geq \kappa_{i} \geq \ldots>0$ tending to zero with a rate $\kappa_{i} \sim 1 / i$ as $i \rightarrow \infty$, for the Sobolev space $X:=H^{1}(0,1)$ under consideration in this section the ill-posedness degree grows at least by one (cf. Hofmann and Tautenhahn [10]) compared to the $L^{2}(0,1)$ case of Section 2. Thus, for a compactification in $H^{1}(0,1)$ 'stronger' restrictions on the admissible solutions $x$ are necessary. However, our aim in this section is also stronger, namely to obtain convergence of approximate solutions $x_{\eta}$ to $x^{*}$ in the $H^{1}(0,1)$-norm (3.1).

Here we consider, for given constants $a_{1}, a_{2}, b_{1}, b_{2}$ and $c$ with

$$
0<a_{1}<b_{1}, a_{2}<b_{2},
$$

the domain

$$
D:=\left\{x:[0,1] \rightarrow\left[a_{1}, b_{1}\right], \begin{array}{c}
\exists x^{\prime}:[0,1] \rightarrow\left[a_{2}, b_{2}\right], \quad x^{\prime} \text { left-continuous for } t \in(0,1], \\
T\left(x^{\prime}\right) \leq c,
\end{array} \quad x^{\prime} \text { right-continuous for } \mathrm{t}=0,\right\},
$$

where the function $x^{\prime}(t)(0 \leq t \leq 1)$ a.e. in $[0,1]$ coincides with a derivative of $x(t)$ in the classical sense. Obviously we get $D \subset H^{1}(0,1)$ and hence every function $x \in D$ with $D$ from $(3.3)$ - (3.4) is continuous. In analogy to Lemma 2.3 we have in the Sobolev space case:

Lemma 3.1 The domain $D$ from (3.3) - (3.4) is a compact subset of $H^{1}(0,1)$ with $D \subset D_{0}^{+}$.

In contrast to the $L^{p}$-case the restriction of the total variation, here $T\left(x^{\prime}\right) \leq c$, is only needed to show the compactness of the domain $D$. It has no relevance for the convergence of the images $F\left(x_{\eta}\right)$ of approximate solutions $x_{\eta}$ to $F\left(x^{*}\right)$ in $L^{2}(0,1)$ as $\eta$ tends to zero. 
The discretization of the autoconvolution problem $(1.1)-(1.3)$, where the operator $F$ from (1.3) maps in the form

$$
F: D \subset H^{1}(0,1) \rightarrow L^{2}(0,1)
$$

and where the domain $D$ is defined by $(3.3)$ - (3.4) will be performed similar to the $L^{p}(0,1)$ case. However, piecewise constant functions are not in $H^{1}(0,1)$. Therefore, we use continuous piecewise linear approximate functions. Here, let (in contrast to Section 2)

$$
t_{j}:=j h \quad(j=0, \ldots, n)
$$

denote the $n+1$ nodes subdividing the interval $[0,1]$, and again $I_{j}=((j-1) h, j h]$. Furthermore, the $x_{j}$ again denote approximate values of $x\left(t_{j}\right)$. As the discrete autoconvolution operator we introduce here:

$$
\underline{F}: \mathbb{R}^{n+1} \rightarrow \mathbb{R}^{n}
$$

where $\underline{F}(\underline{x})=\left(z_{1}, \ldots, z_{n}\right)^{T}$ and for $i=1,2, \ldots, n$ :

$z_{i}=\int_{0}^{i h}\left(E_{1}(\underline{x})\right)(i h-t)\left(E_{1}(\underline{x})\right)(t) d t=\sum_{j=1}^{i} \frac{h}{6}\left(2 x_{i-j} x_{j}+x_{i-j+1} x_{j}+x_{i-j} x_{j-1}+2 x_{i-j+1} x_{j-1}\right)$.

By $E_{1}: \mathbb{R}^{n+1} \rightarrow H^{1}(0,1)$ we denote in contrast to Section 2 the operator of piecewise linear interpolation according to

$$
\left(E_{1}(\underline{x})\right)(t):=\frac{t-j h}{h}\left(x_{j}-x_{j-1}\right)+x_{j} \quad\left(t \in I_{j}, j=1, \ldots, n\right) .
$$

For noisy data (see (2.15)) we search for a minimizer

$$
\underline{x}^{o p t}=\left(x_{0}^{o p t}, x_{1}^{o p t}, \ldots, x_{n}^{o p t}\right)^{T}
$$

of the least-squares problem (2.20) with $M$ from

$$
M:=\left\{\underline{x} \in \mathbb{R}^{n+1}: \begin{array}{c}
0<a_{1} \leq x_{i} \leq b_{1}(i=0, \ldots, n), \\
h a_{2} \leq x_{i}-x_{i-1} \leq h b_{2}(i=1, \ldots, n),
\end{array} \quad \sum_{i=1}^{n-1}\left|x_{i+1}-2 x_{i}+x_{i-1}\right| \leq h c\right\} .
$$

With the same arguments as before it follows that (2.20) is solvable. The choice of $\underline{F}$ is due to the fact that we have to guarantee the validity of formula (2.24) with $F_{\eta}$ from (2.23).

By setting for the approximate solution

$$
x_{\eta}:=E_{1}\left(\underline{x}^{o p t}\right),
$$


where $\eta=(\delta, h)^{T}$, we also have $x_{\eta} \in D$ with $D$ according to (3.3)-(3.4). Moreover, it can be shown that as in Lemma 2.7 we have $\left\|F\left(x_{\eta}\right)-F\left(x^{*}\right)\right\|_{L^{2}(0,1)} \rightarrow 0$ for $\eta \rightarrow 0$. The proof dealing with the $H^{1}(0,1)$ approximation of functions by linear splines is omitted here. Using again Lemma 2.4 with $X:=H^{1}(0,1)$ and $Y:=L^{2}(0,1)$ we obtain:

Theorem 3.2 Consider the autoconvolution problem (1.1) - (1.3) with $D(F):=D$ from (3.3) - (3.4) and denote by $x^{*} \in D$, for given right-hand side $y^{*} \in F(D(F))$, the unique solution of the autoconvolution equation. Then the family of approximate solutions $x_{\eta}$ converges to the solution $x^{*}$ of (1.2):

$$
\left\|x_{\eta}-x^{*}\right\|_{H^{1}(0,1)} \rightarrow 0 \quad \text { as } \quad \eta \rightarrow 0 .
$$

\section{Monotonicity Constraints}

In this section we deal with solutions of the autoconvolution equation subject to the set of monotone and uniformly bounded functions considered as a particular subset of the functions possessing a bounded total variation.

First we consider the domain

$$
D:=\{x: 0 \leq x(t) \leq b, t \in[0,1], x \text { non-increasing }\}
$$

forming a compact subset in $L^{p}(0,1), 1 \leq p<\infty$. Then the operator $F$ from (2.10) is also injective, since $D \subset D_{0}^{+} \cup\{0\}$ and $x(t)=0(0 \leq t \leq 1)$ is the only function of $D$ according to $(4.1)$ with $x(0)=0$. The discretization of this monotonicity case is completely the same as given in Section 2 for the total variation case with the exception of the fact that we have to introduce

$$
M:=\left\{\underline{x} \in \mathbb{R}^{n}: 0 \leq x_{n} \leq \ldots \leq x_{1} \leq b\right\} .
$$

replacing (2.21). Since each monotone function is of bounded variation, we obtain the convergence results of Section 2 with $c=b$ and $a=0$.

Now we change to the case of non-decreasing solutions, where

$$
D:=\{x: 0 \leq x(t) \leq b, t \in[0,1], x \text { non-decreasing }\}
$$

and

$$
M:=\left\{\underline{x} \in \mathbb{R}^{n}: 0 \leq x_{1} \leq \ldots \leq x_{n} \leq b\right\} .
$$


The set $D$ from (4.3) is also compact in $L^{p}(0,1)$, but the injectivity of $F$ fails (cf. [8]). Because of that we have to distinguish two cases:

On the one hand let $y \in R_{0}^{+}$, i.e. $y(s)>0$ if $s>0$. Then the corresponding solution $x^{*}(t)$ is uniquely determined from $y$ a.e. in $[0,1]$ and $\left\|F\left(x_{\eta}\right)-F\left(x^{*}\right)\right\|_{L^{2}(0,1)} \rightarrow 0$ for $\eta \rightarrow 0$ also implies $\left\|x_{\eta}-x^{*}\right\|_{L^{p}(0,1)} \rightarrow 0$, since Tikhonov's lemma (see Lemma 2.4) in fact only needs the local injectivity condition $F(x)=F\left(x^{*}\right)(x \in D) \Longrightarrow x=x^{*}$.

On the other hand, let $y \in R_{\varepsilon}^{+}$for $\varepsilon>0$, i.e. $y(s)=0$ if $s \in[0, \varepsilon]$. As shown in [8], in such a case the autoconvolution operator $F$ is non-injective and it holds:

$$
x^{*}(t)=\left\{\begin{array}{cc}
0 & \text { a.e. in }\left[0, \frac{\varepsilon}{2}\right] \\
\text { uniquely determined } & \text { a.e. in }\left[\frac{\varepsilon}{2}, 1-\frac{\varepsilon}{2}\right] . \\
\text { arbitrarily non-negative } & \text { in }\left[1-\frac{\varepsilon}{2}, 1\right]
\end{array}\right.
$$

Consequently, we have $x^{*} \in D_{\frac{\varepsilon}{2}}^{+}$. Since the values $x^{*}(t)$ do not depend on $y$ for $t \in\left[1-\frac{\varepsilon}{2}, 1\right]$, we cannot expect any information about the solution in this subinterval from the data. Therefore, it makes sense to solve the equation (1.1) only on the interval $\left[\frac{\varepsilon}{2}, 1-\frac{\varepsilon}{2}\right]$. We will show that this case is reducible to the already treated case $y \in R_{0}^{+}$. Because of this we define the operator $F_{\varepsilon}: L^{p}\left(\frac{\varepsilon}{2}, 1-\frac{\varepsilon}{2}\right) \rightarrow L^{2}(\varepsilon, 1)$ as

$$
\left[F_{\varepsilon}(x)\right](s):=\int_{\frac{\varepsilon}{2}}^{s-\frac{\varepsilon}{2}} x(s-t) x(t) d t .
$$

Then we have $[F(x)](s)=\left[F_{\varepsilon}(x)\right](s)$ for $\frac{\varepsilon}{2} \leq s \leq 1-\frac{\varepsilon}{2}$. By using the transformations

$$
\tilde{t}:=\frac{t-\frac{\varepsilon}{2}}{1-\varepsilon}, \quad \tilde{s}:=\frac{s-\varepsilon}{1-\varepsilon}
$$

and

$$
\tilde{x}(\tilde{t}):=x\left((1-\varepsilon) \tilde{t}+\frac{\varepsilon}{2}\right)=x(t), \quad \tilde{y}(\tilde{s}):=y((1-\varepsilon) \tilde{s}+\varepsilon)=y(s), \quad \tilde{F}_{\varepsilon}(\tilde{x}):=F_{\varepsilon}(x),
$$

we obtain an operator $\tilde{F}_{\varepsilon}: L^{p}(0,1) \rightarrow L^{2}(0,1)$ defined by

$$
\left[\tilde{F}_{\varepsilon}(\tilde{x})\right](\tilde{s}):=(1-\varepsilon) \int_{0}^{\tilde{s}} \tilde{x}(\tilde{s}-\tilde{t}) \tilde{x}(\tilde{t}) d \tilde{t}
$$

Then we get $\tilde{x} \in L^{p}(0,1)$ if $x \in L^{p}(0,1)$, and instead of (1.2) we have to solve the equation $\tilde{F}_{\varepsilon}(\tilde{x})=\tilde{y}$ now. From $y \in R_{\varepsilon}^{+}$and $x \in R_{\frac{\varepsilon}{2}}^{+}$it follows that $\tilde{y} \in R_{0}^{+}$and $\tilde{x} \in D_{0}^{+}$, respectively. Hence we have $\tilde{F}_{\varepsilon}(\tilde{x})=(1-\varepsilon) F(\tilde{x})$ for all $\tilde{x} \in D_{0}^{+}$. Therefore, we can proceed as in the injective case and compute converging approximate solutions $\tilde{x}_{\eta}$. Then we transform back to the interval $\left[\frac{\varepsilon}{2}, 1-\frac{\varepsilon}{2}\right]$ and obtain approximate solutions with satisfactory properties on 
this interval, where the performed linear transformation retains the monotonicity. Finally we extend the solution by zero on the interval $\left[0, \frac{\varepsilon}{2}\right)$. On the other remaining subinterval $\left(1-\frac{\varepsilon}{2}, 1\right]$ the solution can be extended arbitrarily provided that the monotonicity requirement is satisfied. Unfortunately, the value of $\varepsilon$ is unknown if only discrete noisy data are given. In some situations, however, this value can be estimated and the transformation procedure becomes applicable.

\section{$5 \quad$ Numerical Examples}

In the concluding section we present some case studies on the behaviour of approximate discrete least-squares solutions to the autoconvolution equation (1.1) from noisy data, where we follow the approach of Section 2.

The first study is devoted to the case of a continuous, but non-monotone exact solution. We use the example

$$
x^{*}(t)=-3 t^{2}+3 t+\frac{1}{4}=1-3\left(t-\frac{1}{2}\right)^{2} \quad(0 \leq t \leq 1)
$$

with the right-hand side

$$
y^{*}(s)=\frac{3}{10} s^{5}-\frac{3}{2} s^{4}+s^{3}+\frac{3}{4} s^{2}+\frac{1}{16} s \quad(0 \leq s \leq 1)
$$

and obtain $a:=0.25 \leq x^{*}(t) \leq b:=1, T\left(x^{*}\right)=1.5$ and $x^{*} \in D$ with $D$ from (2.5) - (2.6). The noisy data $\underline{\hat{y}}$ were generated by adding normally distributed pseudorandom numbers with zero mean and standard deviation $\sigma y_{i}$ ( $\sigma$ fixed) to the discrete values $y_{i}$ of (5.2). We used varying values $c$ as upper bounds for the total variation of the discretized solutions.

The nonlinear optimization problem (2.20) was numerically solved by a Gauss-Newton code. In the case of unacceptable Gauss-Newton steps this code uses the Marquardt method. The theory of this procedure is due to [3, pp. 348-368] (for the algorithm see [3, pp. 369-383]). We used penalty terms to handle the constraints of $D$. In all figures presented below the solid lines give the exact solution $x^{*}$ according to (5.1), whereas the lines with small circles express the approximate solutions $x_{\eta}$ such that every circle corresponds to a grid point of discretization.

In the Figures 1 and 2 we compare approximate solutions $x_{\eta}$ in the case of unperturbed data $(\sigma=0)$ using $n=50$ grid points and different bounds $c$ for the total variation. For an 
appropriate choice $c=1.5$ associated with the really arising total variation level, the approximate solution is very good in the noiseless case (see Figure 1), whereas an underestimated value $c=0.8<T\left(x^{*}\right)$ corresponds to an overregularized solution (see Figure 2), which is much too 'flat' compared to the function $x^{*}$ to be determined.

Now we turn to the case of noisy data. For all computations in the context of the Figures $3-6$ a per mille noise level $\sigma=10^{-3}$ was used. We begin with a situation (see Figure 3$)$, where the total variation bound was omitted $(c=\infty)$. Then the set $M$ of admissible discrete solutions contains strongly oscillating vectors. Especially for $t$ from the right halfinterval of $[0,1]$ the quality of the approximate solutions may be very bad in that situation.

The Figure 4 illustrates in a rather convincing manner the utility of the total variation approach presented above in Section 2 for handling noisy data. In particular, the approximation quality of $x_{\eta}$ in Figure 4 with $c=1.5$ at the right end of the interval is much better than in Figure 3. We can motivate this right-end effect as follows: By the autoconvolution of a function $x(t)(0 \leq t \leq 1)$ the values $x(t)$ for small $t$ influence the function values $y(s)$ in some sense more than the values $x(t)$ with $t$ close to 1 . Namely, $x(t)$ only influences $y(s)$ for $s>t$. As a consequence, the reconstruction of $x(t)$ from $y$ is more stable for smaller $t$, since then the function $y(s)=[x * x](s)(0 \leq s \leq 1)$ has collected more information about the value $x(t)$ to be determined. In the case of overregularization $(c$ is selected too small compared to $T\left(x^{*}\right)$ ), this phenomenon may cause large reconstruction errors specifically at the right end of the interval $[0,1]$ (see Figure 5 with $c=1.0$ ).

We should mention that the analysis of the problem based on Lemma 2.4 does not provide any rate of convergence for the solution error $\left\|x_{\eta}-x^{*}\right\|_{L^{p}(0,1)}$ depending on $\left\|F\left(x_{\eta}\right)-F\left(x^{*}\right)\right\|_{L^{2}(0,1)}$. On the other hand, Lemma 2.7 shows that the order of magnitude for the discrepancy norm $\left\|F\left(x_{\eta}\right)-F\left(x^{*}\right)\right\|_{L^{2}(0,1)}$ corresponds with the maximum $\max \left(h \bar{b}^{2}, h \bar{b} \bar{c}, \delta\right)$, where $h:=1 / n, \bar{b}:=\sup _{\substack{x \in D \\ t \in[0,1]}} x(t)$ and $\bar{c}:=\sup _{\substack{x \in D \\ t \in[0,1]}} T(x)$. For sufficiently large $n$ this discrepancy norm is dominated by the noise level $\delta$, or in our case study by the value $\sigma$. So we can see comparing the Figures 4 and 6 that different discretization levels $n=50$ and $n=25$ yield approximate solutions with nearly the same accuracy provided that the noise level $\left(\sigma=10^{-3}\right)$ does not change.

To a second study we have been motivated by numerical experiments carried out by Kutsche in her thesis [11]. There it was shown that the constraint of bounded variation is very useful in the $L^{1}$-approximation of piecewise continuous solutions to Abel integral 
equations. We now will demonstrate the effects of using the least-squares method under total variation constraints in the case of non-smooth functions possessing jumps. Therefore we consider as the exact solution the step function

$$
x^{*}(t)=\left\{\begin{array}{cc}
0.5 & \text { if } 0 \leq t \leq 0.5 \\
0.25 & \text { if } 0.5<t \leq 0.8 \\
0.75 & \text { if } 0.8<t \leq 1
\end{array}\right.
$$

with the right-hand side

$$
y^{*}(s)=\left\{\begin{array}{cc}
0.25 t & \text { if } 0 \leq t \leq 0.5 \\
0.125 & \text { if } 0.5<t \leq 0.8 \\
0.5 t-0.275 & \text { if } 0.8<t \leq 1
\end{array}\right.
$$

The exact solution $x^{*}$ is discontinuous, non-monotone but a function of bounded variation. Its total variation can easily computed as $T\left(x^{*}\right)=0.75$. The function $x^{*}$ is bounded, positive and left-continuous on the whole interval $[0,1]$. Therefore the requirements of the set $D$ from (2.5) - (2.6) are fulfilled.

We will now compare the approximate solutions of this example for different choices of the parameter $c$. Let the number of discretization points $n=50$ and the value $\sigma=10^{-2}$ of noise be constant throughout this study. Then we are able to control the solution by changing the parameter $c$.

In the Figures 7 - 10 the graphs of both the numerical solution $x_{\eta}$ and the exact solution $x^{*}$ (bold line) are drawn as piecewise constant functions. In our first example (Figure 7) we computed the solution without any total variation restriction $(c=\infty)$. The solution is - as in the first example - rather bad and highly oscillating. However, it is to mention that the jumps of $x^{*}$ are reconstructed relatively good in this case. In Figure 8 the situation $c=T\left(x^{*}\right)=0.75$ is illustrated. Here the solution is much smoother than in the unconstrained case, but the points with maximal approximation errors are now the jumps at $t=0.5$ and $t=0.8$. In these points the approximate solution is 'oversmoothed'. This depends on the fact that the smoothing effect of regularization acts uniformly on the whole interval $[0,1]$, but the character of a jump function does not correspond with this property. Therefore the jumps are blurred by that choice of $c$. Moreover, the 'right-end effect' discussed above is superposed and leads to growing errors near $t=1$.

Finally, in Figure 9 with $c=0.5$ and Figure 10 with $c=2$ we demostrate two more situations. If $c$ underestimates the value $T\left(x^{*}\right)$, then the effect of blurring the jumps is still 
more pronounced. On the other hand, the admissible oscillation level grows if $c$ overestimates $T\left(x^{*}\right)$. In that case, however, the location of jumps can be determined rather precise. That means, if one supposes that the exact solution is a step function, then it is recommended to choose $c$ not too small. This allows some oscillations around the exact solution whose amplitudes are small if $c$ is not chosen much too large.

\section{References}

[1] AcAR, R.; Vogel, C.R.: Analysis of total variation penalty methods. Inverse Problems 10 (1994), 1217-1229.

[2] Baumeister, J.: Stable Solution of Inverse Problems. Vieweg, Braunschweig 1987.

[3] Dennis, J.E.; Gay, D.M.; Welsch, R.E.: An adaptive nonlinear least-squares algorithm. ACM Trans. Math. Softw. 7 (1981), 348-368.

[4] Engl, H.W.; Hofmann, B.; Zeisel, H.: A decreasing rearrangement approach for a class of ill-posed nonlinear integral equations. Journal of Integral Equations and Applications 5 (1993), 443-463.

[5] Fleischer, G; Hofmann, B.: On inversion rates for the autoconvolution equation. Inverse Problems 12 (1996), 419-435.

[6] Gellrich, C.; Hofmann, B.: A study of regularization by monotonicity. Computing 50 (1993), 105-125.

[7] Gorenflo, R.: Computation of rough solutions of Abel integral equations. In: Inverse and Ill-Posed Problems (Eds.: H.W. Engl, C.W. Groetsch), Academic Press, Boston, 1987, 195-210.

[8] Gorenflo, R.; Hofmann, B.: On autoconvolution and regularization. Inverse Problems 10 (1994), 353-373.

[9] Hofmann, B.: Regularization for Applied Inverse and Ill-Posed Problems. B.G. Teubner, Leipzig 1986.

[10] Hofmann, B.; Tautenhahn, U.: On ill-posedness measures and space change in Sobolev scales. Paper submitted. 
[11] Kutsche, C.: Produktquadraturverfahren für nicht-glatte Lösungen Abelscher Integralgleichungen erster und zweiter Art. Dissertation, Freie Universität Berlin, FB Mathematik und Informatik, Berlin 1994.

[12] Natanson, I.P.: Theorie der Funktionen einer reellen Veränderlichen. AkademieVerlag, Berlin, 1969.

[13] Tikhonov, A.N; Goncharsky, A.V.; Stepanov, V.V.; Yagola, A.G.: Regularizing algorithms and a priori information (in Russian). Nauka, Moscow, 1983. 


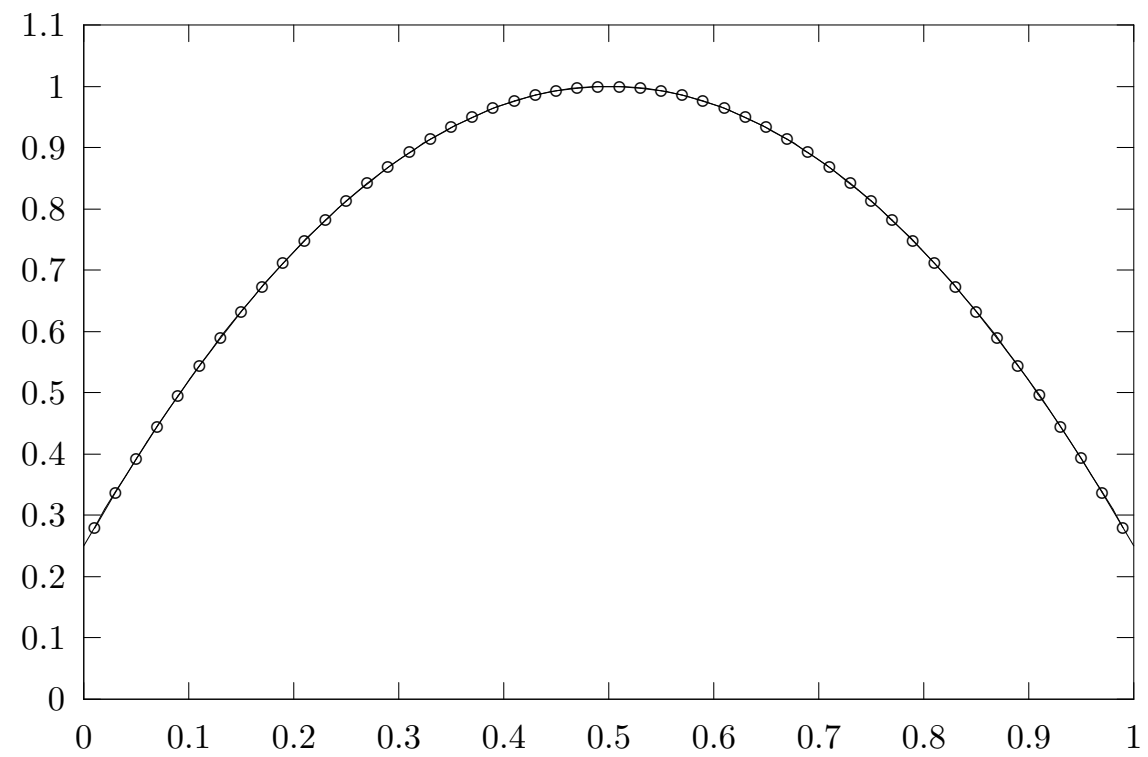

Figure 1: Least-squares solution of $\underline{F}(\underline{x})=\underline{\hat{y}}, \sigma=0, n=50, c=1.5$

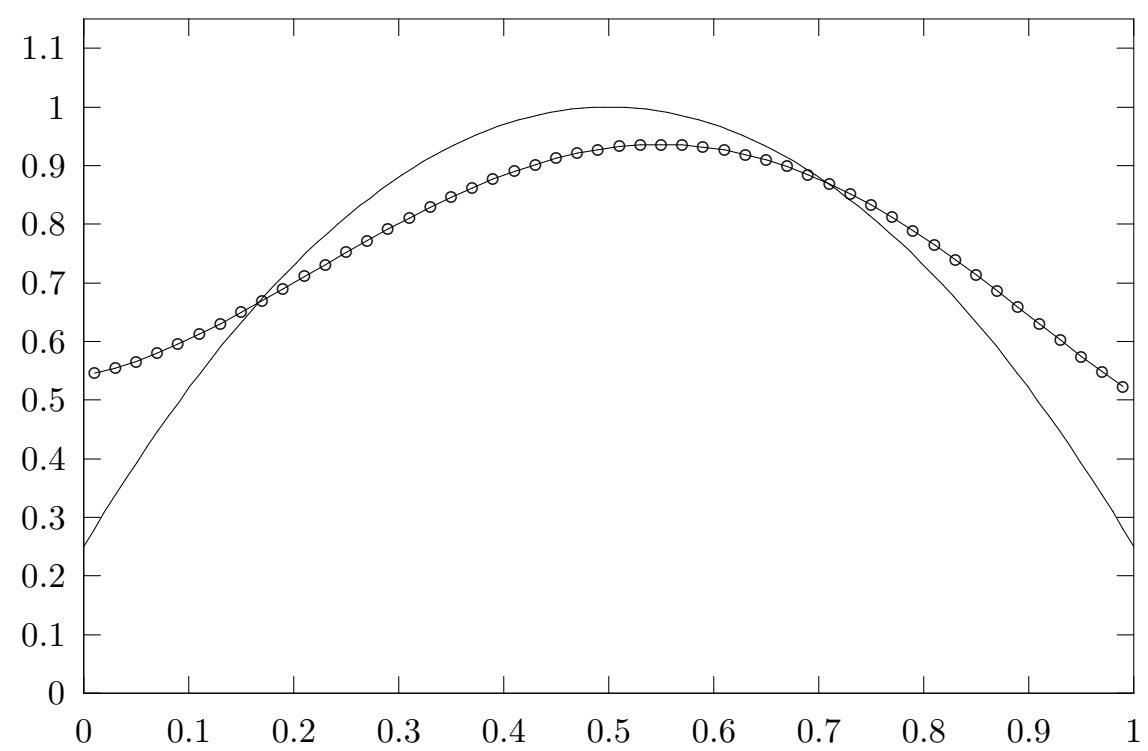

Figure 2: Least-squares solution of $\underline{F}(\underline{x})=\underline{\hat{y}}, \sigma=0, n=50, c=0.8$ 


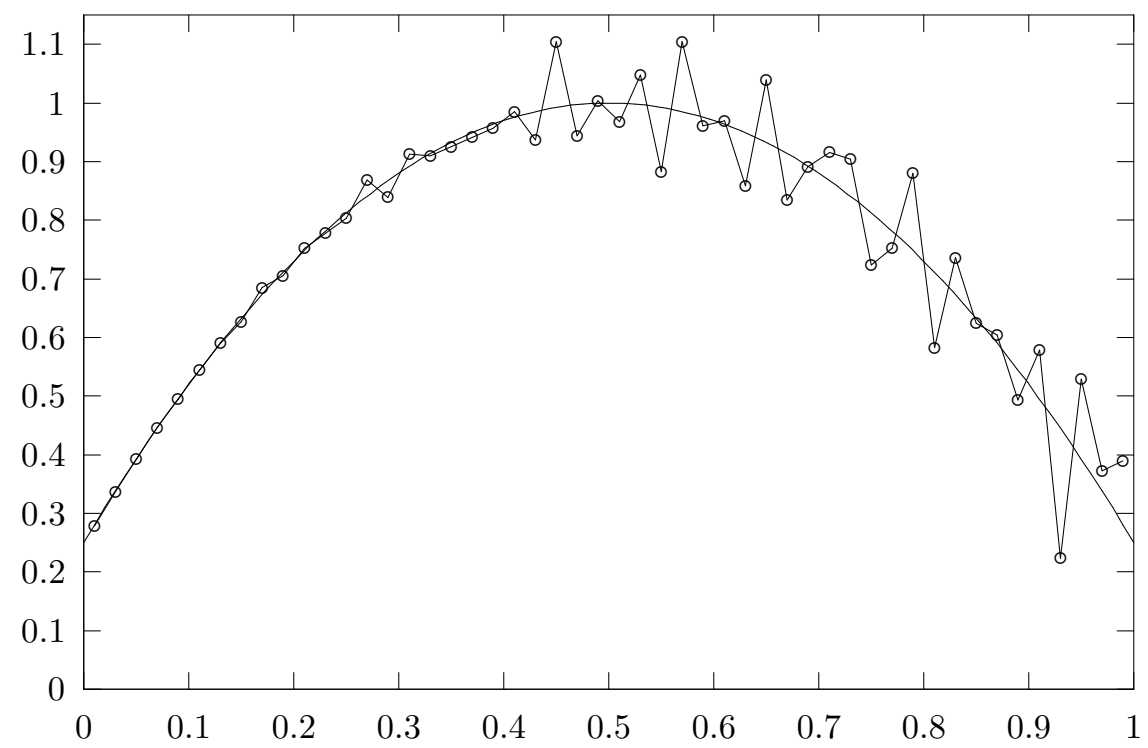

Figure 3: Least-squares solution of $\underline{F}(\underline{x})=\underline{\hat{y}}, \sigma=10^{-3}, n=50, c=\infty$

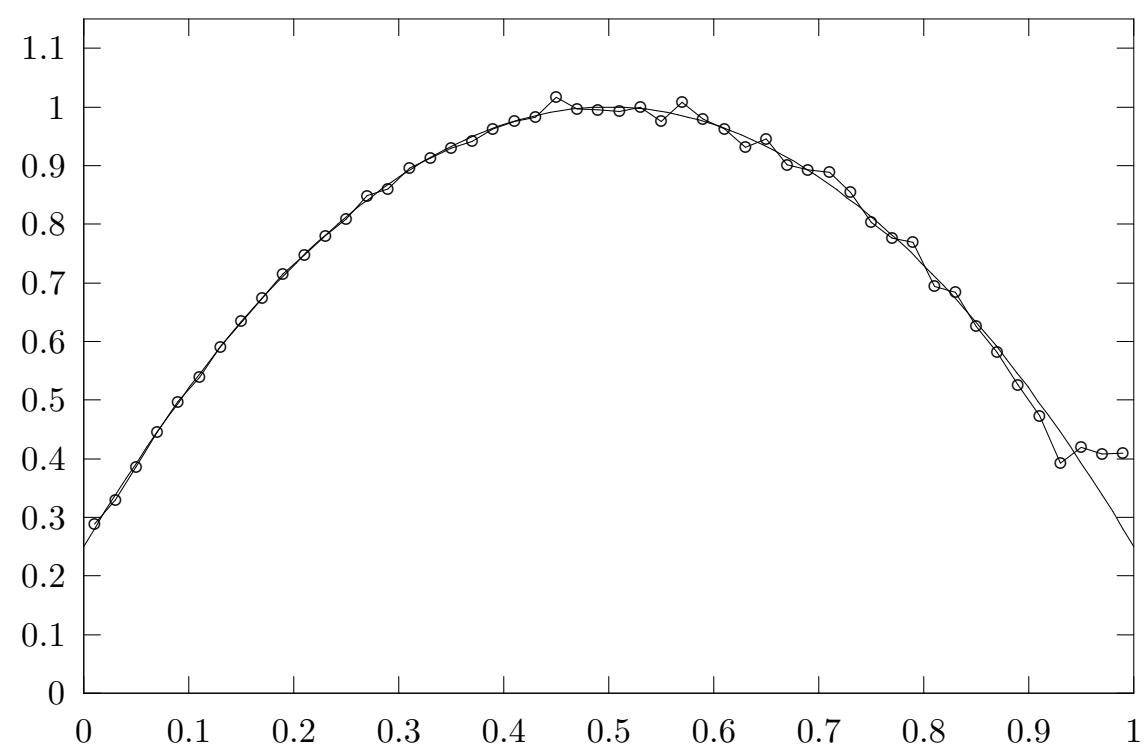

Figure 4: Least-squares solution of $\underline{F}(\underline{x})=\underline{\hat{y}}, \sigma=10^{-3}, n=50, c=1.5$ 


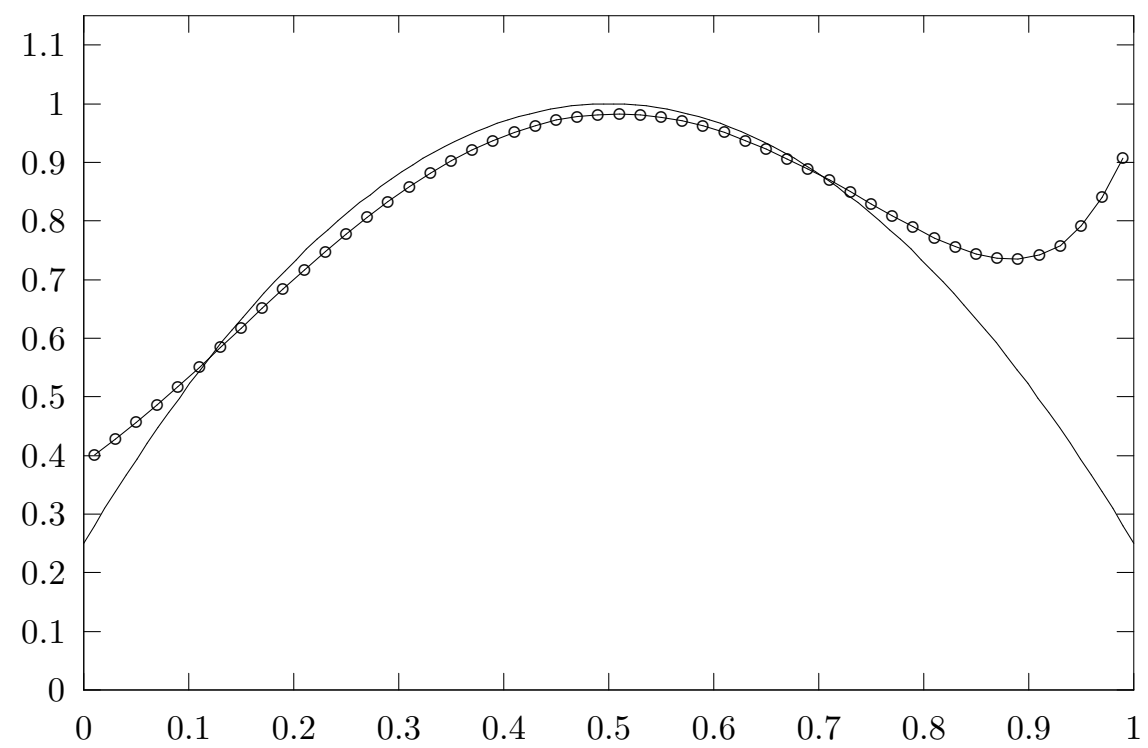

Figure 5: Least-squares solution of $\underline{F}(\underline{x})=\underline{\hat{y}}, \sigma=10^{-3}, n=50, c=1.0$, (inappropriate initial values used in Gauss-Newton-method)

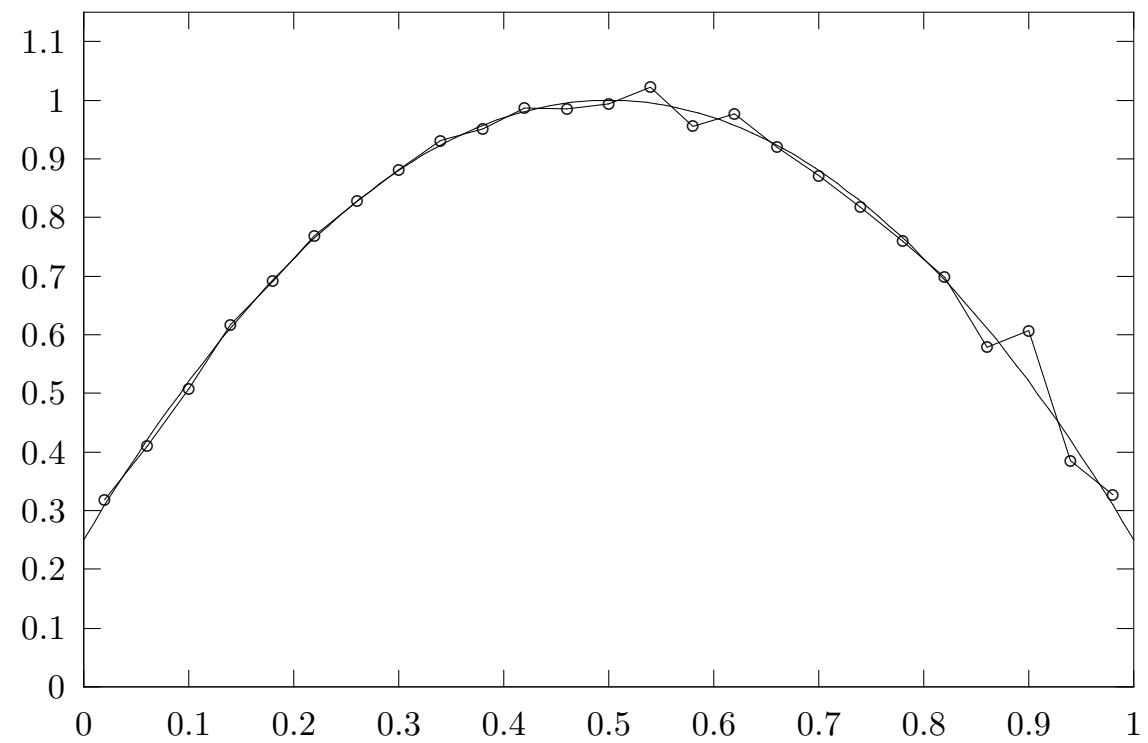

Figure 6: Least-squares solution of $\underline{F}(\underline{x})=\underline{\hat{y}}, \sigma=10^{-3}, n=25, c=1.5$ 


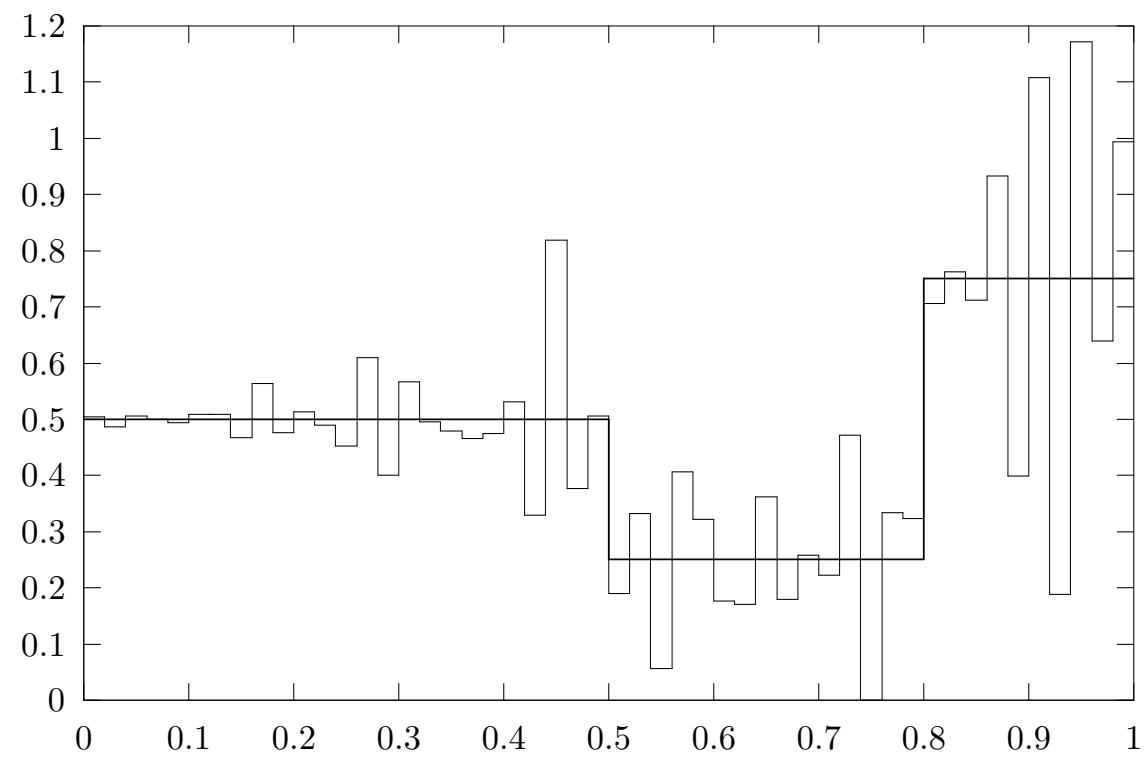

Figure 7: Least-squares solution of $\underline{F}(\underline{x})=\underline{\hat{y}}, \sigma=10^{-2}, n=50, c=\infty$

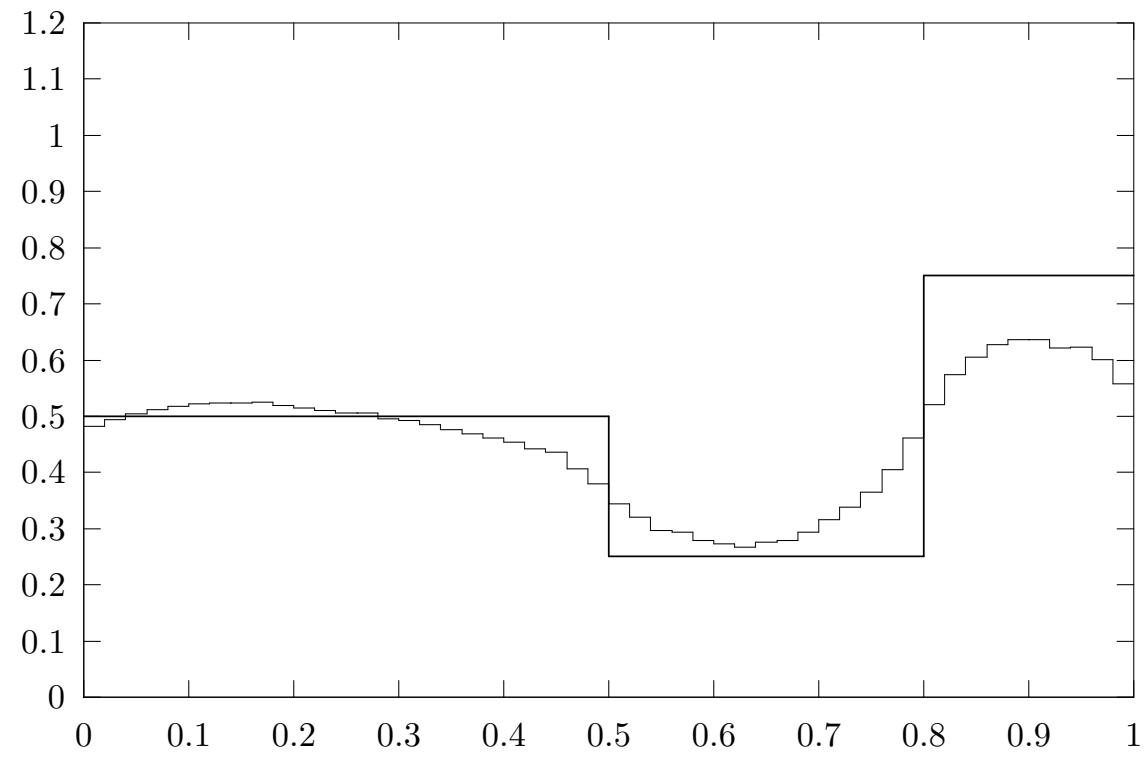

Figure 8: Least-squares solution of $\underline{F}(\underline{x})=\underline{\hat{y}}, \sigma=10^{-2}, n=50, c=0.75$ 


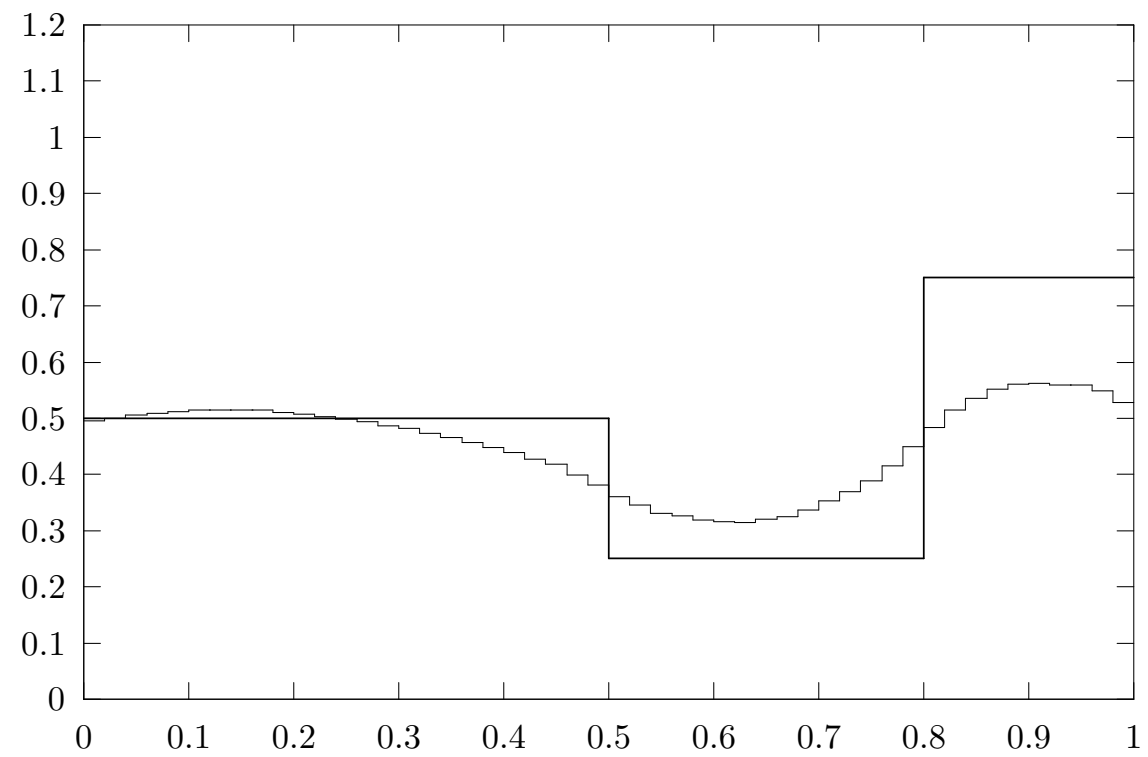

Figure 9: Least-squares solution of $\underline{F}(\underline{x})=\underline{\hat{y}}, \sigma=10^{-2}, n=50, c=0.5$

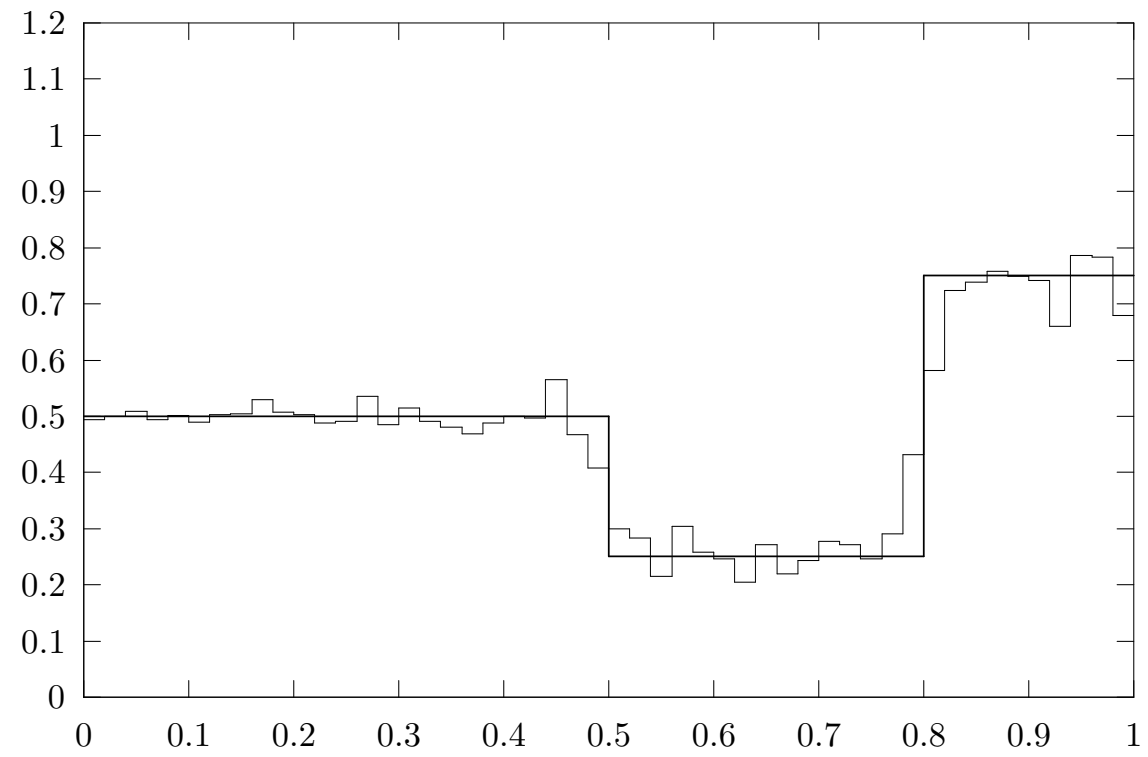

Figure 10: Least-squares solution of $\underline{F}(\underline{x})=\underline{\hat{y}}, \sigma=10^{-2}, n=50, c=2.0$ 